Anales de Geografía de la Universidad Complutense ISSN: 0211-9803

http://dx.doi.org/10.5209/AGUC.69337

\title{
Repercusiones de las condiciones ambientales altitudinales en la variabilidad de la disponibilidad hídrica en la cuenca del río Ródano en escenarios de cambio climático
}

\author{
Pedro Pérez-Cutillas ${ }^{1}$; Alfredo Pérez-Morales ${ }^{2}$; Salvador Gil-Guirado ${ }^{3}$ \\ Recibido: 20 de enero del 2018 / Enviado a evaluar: 6 de junio de 2018 / Aceptado: 9 de marzo del 2020
}

Resumen. La cuenca del Ródano se enfrenta a corto y medio plazo a importantes descensos en el volumen de sus aguas y a cambios en la estacionalidad del régimen hidrológico, lo que generará importantes repercusiones económicas y ambientales. El análisis llevado a cabo en el presente artículo emplea un modelo hidrológico sobre valores climáticos del periodo actual que sirve de referencia para la comparación de cuatro escenarios futuros de Concentración de Gases de Efecto Invernadero (RCP). Los resultados demuestran que se puede producir un importante descenso de la capacidad de producción hídrica de la cuenca, llegando en las condiciones más desfavorables al $30 \%$ del total. La comparación de estos efectos en diversos sistemas ambientales caracterizados por rasgos altitudinales, señala importantes diferencias en el balance hídrico final, siendo las áreas de alta montaña las zonas de la cuenca más sensibles al cambio climático. En conclusión, la evidente pérdida de recursos hídricos supone importantes desafíos y obliga a revisar los actuales sistemas de gestión del agua.

Palabras clave: Balance hídrico; modelización; procesos hidrológicos; políticas hidrológicas sostenibles.

\section{[en] Effects of altitudinal environmental conditions on the variability of water yield in the Rhone river basin in climate change scenarios}

Abstract. In the short and medium term, Rhone basin will have significant discharge water decreases and changes in the seasonality of the hydrological regime, causing serious economic and environmental repercussions. The analysis is carried out through a hydrological model applied to the climatic values of observed period, used as reference in the comparison of two Representative Concentration Pathways

1 Grupo de Geografía Física del Departamento de Geociencias de la Universidad de Friburgo (Suiza). Y, Grupo de Erosión y Conservación de Suelos, Centro de Edafología y Biología Aplicada del Segura - Consejo Superior de Investigaciones Científicas (CEBAS-CSIC), Murcia (España).

E-mail: perezcutillas@cebas.cisc.es

2 Departamento de Geografía, Universidad de Murcia (España).

3 Departamento de Geografía, Universidad de Murcia (España). 
(RCPs) scenarios in two future periods. Results have determined a significant decrease in water discharge capacity, being in some cases $30 \%$. Environmental systems characterized by altitudinal features have established important differences in the final water balance, high mountain areas have been the most sensitive areas to climate change. Decrease in water resources will establish important adaptations to the new challenges imposed by these water scarcity scenarios.

Keywords: Water balance; modeling; hydrological processes; sustainable water policies.

[fr] Effets des conditions environnementales altitudinales sur la variabilité de la disponibilité de l'eau dans le bassin du Rhône dans les scénarios de changement climatique

Résumé. À court et moyen terme, le bassin du Rhône subit des baisses importantes du volume de ses eaux et des changements dans la saisonnalité du régime hydrologique, générant d'importantes répercussions économiques et environnementales. L'analyse est réalisée à travers un modèle hydrologique appliqué sur les valeurs climatiques de la période actuelle. Ces données serviront de référence dans la comparaison de deux scénarios de concentration de gaz à effet de serre (PCR) dans deux périodes futures. Les résultats ont déterminé une diminution significative de la capacité de production d'eau du bassin, en supposant dans certains cas 30\% du total. Les analyses élaborées dans les systèmes environnementaux caractérisés par des caractéristiques altitudinales ont établi des différences importantes dans le bilan hydrique final, montrant les zones de haute montagne comme les zones les plus sensible au changement climatique. La perte de ressources en eau impliquera d'importantes adaptations aux nouveaux défis imposés par ces scénarios de rareté croissante de l'eau.

Mots clés: Bilan hydrique; modélisation; processus hydrologiques; politiques hydrologiques durables.

Cómo citar. Pérez-Cutillas, P., Pérez-Morales, A. y Gil-Guirado, S. (2020): Repercusiones de las condiciones ambientales altitudinales en la variabilidad de la disponibilidad hídrica en la cuenca del río Ródano en escenarios de cambio climático. Anales de Geografía de la Universidad Complutense, 40(1), 159-182.

Sumario. 1. Introducción. 2. Área de estudio. 3. Metodología. 3.1. Descripción del modelo "Water Yield". 3.2. Datos de entrada al modelo. 3.2.1. Coberturas del suelo. 3.2.2. Delimitación de cuencas. 3.2.3. Datos del suelo. 3.2.4. Datos climáticos. 3.3. Delimitación altitudinal de la Cuenca. 4. Resultados. 4.1. Tendencias de los datos mensuales de precipitación y evapotranspiración de referencia. 4.2. Resultados del modelo "Water Yield". 4.3. Análisis altitudinal de los valores modelizados. 5. Discusión. 6. Conclusiones. 7. Bibliografía.

\section{Introducción}

Los efectos del cambio climático son cada vez más visibles en los sistemas fluviales de todo el mundo (IPCC, 2014). Las predicciones a corto y medio plazo prevén importantes variaciones en el volumen a la baja y estacionalidad de los regímenes hidrológicos. Esta dinámica supondrá el agravamiento de una serie de problemas que se verán acentuados con especial repercusión en los sistemas socioeconómicos y ambientales (Mendizabal et al., 2014; Pavanelli y Capra, 2014). De acuerdo a este nuevo contexto, los impactos esperados se hacen especialmente graves en las cuencas 
que albergan intensas presiones demográficas y altos desarrollos de producción económica, como es la cuenca del río Ródano (Bréthaut y Clarvis, 2015).

La cuenca del Ródano alberga numerosas actividades socio-económicas con funcionamientos y operatividad estrechamente vinculadas al de sus cursos fluviales, lo que ha organizado una competencia entre los diferentes usuarios de esos recursos hídricos para asegurar sus necesidades. Al tratarse de un conflicto de usos complejo, se ha desarrollado una estrategia de aprovechamiento continuada por las diferentes administraciones competentes durante décadas, sin embargo, las crecientes preocupaciones ambientales están modificando las posturas de uso de los actores involucrados (Bréthaut y Pflieger, 2015). Entre otros, los cambios observados en su régimen hidrológico están obligando a modificar las líneas de gestión en la cuenca del río, políticas que alteran la preferencia de los diferentes usos del agua, especialmente, en los planes de inundaciones, de protección ambiental, y los programas energéticos.

Respecto a las políticas energéticas, están evolucionando en el sentido de la sostenibilidad para favorecer las fuentes de producción renovables y la producción de energía hidroeléctrica en particular (Tonka, 2014). Este cambio se refleja en el refuerzo de las ordenanzas ambientales favorecidas por los reglamentos de la Unión Europea (Directiva Marco del Agua) y el desarrollo de la legislación de aguas en Francia y Suiza. En el caso francés, influenciado por la liberalización del mercado europeo de producción de electricidad, desarrolló un sistema de concesión a operadores privados por un período aproximado de 90 años, el cual terminará en 2023 (Bréthaut y Clarvis, 2015). De acuerdo a lo anterior, parece por tanto interesante analizar las posibles modalidades de gestión que se realizan en río transfronterizo como el Ródano para adaptarse a las nuevas configuraciones climáticas (RuizVillanueva, et al., 2014)

El Ródano, caracterizado por un régimen pluvio-nival muy caudaloso asociado a fuentes de alimentación hídrica principales localizadas a grandes altitudes. Recibe las aguas de los Alpes, por su margen izquierda, y las del Macizo Central francés y de los Vosgos, a través del Saona, por la derecha. Estas al igual que otras regiones montañosas han sido denominadas como "torres de agua" naturales porque proporcionan los recursos hídricos necesarios para asegurar un módulo elevado que ha afianzado la actividad agrícola, industrial y urbana de sus vegas desde el inicio de su ocupación (Vivrioli and Weingartner, 2008). Sin embargo, estas regiones de gran elevación sobre el nivel del mar, presentan una vulnerabilidad mayor que los ámbitos más bajos a los efectos del cambio climático. Los Alpes europeos es una de las regiones del planeta donde las alteraciones provocadas por el clima son realmente perceptibles en la actualidad (CITA). De acuerdo a la serie de registros, el aumento de las temperaturas, ya ha supuesto un incremento de hasta en $2{ }^{\circ} \mathrm{C}$ desde principios del siglo pasado. Además, las predicciones de los modelos climáticos regionales sugieren que para el año 2100 se podrían alcanzar en Suiza valores térmicos superiores a las actuales con rangos de $3-5{ }^{\circ} \mathrm{C}$ en invierno y de $6-7{ }^{\circ} \mathrm{C}$ en verano, asociados a un aumento de las precipitaciones en invierno y una drástica disminución en el periodo estival (Beniston, 2012). De acuerdo al estado de la ocupación actual, los impactos directos e indirectos afectarán a sectores económicos clave como el turismo, la 
energía hidroeléctrica, la agricultura y a las grandes compañías de seguros que deberán hacer frente a los gastos originados por desastres naturales cada vez más frecuentes (Beniston y Stoffel, 2014).

En lo que se refiere concretamente a la variabilidad de los recursos hídricos, las tendencias mostradas por los estudios de cambio climático (Gobiet et al., 2014) están anunciando eventos más extremos (sequías e inundaciones), una mayor reducción de la superficie ocupada por glaciares y la alteración de las condiciones del deshielo, que están modificando significativamente los diferentes regímenes hidrológicos en la cuenca del Ródano (Beniston et al., 2011; Chauveau et al., 2013). Los probables cambios referentes a la calidad del agua provocadas por un aumento de la temperatura tendrán consecuencias significativas para los ecosistemas acuáticos y ribereños, así como para las infraestructuras dependientes de los sistemas de producción hidroeléctricos y los de refrigeración de las centrales de energía nuclear existentes en la parte francesa de la cuenca (Gaudard et al., 2014; Pellicciotti et al., 2014; Bréthaut y Pflieger, 2015). Asimismo, pero en otro orden de magnitud, la reducción del caudal del Ródano podría suponer un impacto significativo en la salinidad del mar Mediterráneo, como ya se demostró con la reducción del volumen de agua aportada por el Nilo (Rohling y Bryden, 1992; Bethoux y Gentili, 1999).

De acuerdo a lo anterior, la atención y el esfuerzo parece centrarse en los estudios de los recursos hídricos en las regiones montañosas, especialmente, en las áreas donde los cambios en los regímenes hídricos irán acompañados de peligros geomorfológicos más frecuentes (Barboux et al, 2016) relacionados con el aumento de los eventos de precipitación intensa y la fusión de los suelos permanentemente congelados (Delaloye et al., 2010). En este sentido, Proyectos como ACQWA, del Séptimo Programa Marco de la Unión Europea, analizan los impactos del calentamiento global en la cantidad y calidad del agua; y proporcionan herramientas de ayuda para la solucionar cuestiones relacionadas con la gestión actual del agua y los posibles cambios en sus demandas futuras. La aplicación de políticas sólidas que puedan hacer frente a los nuevos escenarios de escasez y uso del agua en las próximas décadas adquieren especial relevancia pues permitirán solucionar de manera más efectiva las posibles rivalidades entre sectores económicos que puedan enfrentarse por los recursos hídricos (Beniston et al., 2011).

A este respecto, la modelización y las proyecciones de los efectos del cambio climático sobre la cuantificación y disponibilidad hídrica son cada vez más necesarias para la mejora y refuerzo en la planificación de estrategias en la gestión del agua. Pese a las bondades de estos instrumentos, conviene no olvidar que sus predicciones no están exentas aún de un grado de incertidumbre asociado a la dispersión de los modelos climático utilizados, a la propia variabilidad climática interna, y a las dificultades para transferir los resultados del modelo climático a escalas espaciales y temporales que requieren los modelos hidrológicos (Fatichi et al., 2014). Por lo tanto, el conocimiento, y la reducción en la medida de lo posible de estas incertidumbres es esencial para poder realizar proyecciones en la estimación de escorrentías (Huss et al., 2014). 
El objetivo principal de este trabajo se centra en analizar los efectos del cambio climático en la capacidad de disponibilidad hídrica en la cuenca del río Ródano, y en establecer las posibles causas de la variación del balance de agua de la cuenca, atendiendo a factores ambientales altitudinales. El estudio se llevó a cabo mediante la aplicación de un modelo de servicios ecosistémicos, a través de su evaluación en cinco escenarios climáticos. El primero de ellos, referido al periodo actual, que servirá de referencia en la comparación de dos escenarios de Concentración de Gases de Efecto Invernadero (RCP) y en dos periodos futuros.

\section{2. Área de estudio}

La justificación del área de estudio del presente trabajo atiende a tres razones principales. La primera está relacionada con la envergadura espacial del su sistema fluvial. El Ródano es uno de los ríos europeos de mayor entidad (con un caudal anual medio de $1700 \mathrm{~m}^{3} / \mathrm{s}$, y una longitud de $810 \mathrm{~km}$ ) y el más importante de la cuenca mediterránea, llegando a suministrar el $20 \%$ del aporte agua fluvial en el Mediterráneo (http://www.ird.fr/). La segunda, se debe al gran interés económico (navegación comercial y producción hidroeléctrica) que existe a lo largo de sus riberas. Y la tercera es, es por su alto valor ecológico (Lamouroux et al., 2013). La combinación de lo anterior, representa un ámbito geográfico lo suficientemente representativo para poner de manifiesto las repercusiones que puedan tener la variabilidad esperada de los caudales circulantes ante los efectos de un posible cambio de escenario climático.

El área de drenaje del Ródano se ubica en los países de Suiza y Francia $(97.800$ $\mathrm{km}^{2}$ ), siendo la mayor parte francesa con una superficie aproximada de $86.500 \mathrm{~km}^{2}$ (Figura 1). Su elevado caudal está alimentado por varias cadenas montañosas, siendo la cordillera de los Alpes y el macizo del Jura las más importantes. Al cauce principal, se unen importantes afluentes como el Saone y el Ain en el norte y el Isere, Ardeche y Durance en el sur, lo que le confieren elevados caudales punta, que en algunos casos han llegado a superar los $11.000 \mathrm{~m}^{3} / \mathrm{s}$ (http://hydro.eaufrance.fr/).

Según Bréthaut y Clarvis (2015), la cuenca del río Ródano se caracteriza por la existencia de diferentes patrones hídricos, que pueden resumirse en tres áreas bien diferenciadas. La primera está situada en el alto Ródano, en los cantones suizos de Valais y Vaud. Cuenta con un régimen de escorrentía nivo-glacial que proporciona una mayor descarga en verano que en invierno. El papel de los glaciares como fuente de alimentación es muy importante en el régimen hidrológico de esta parte del Ródano, ocupando una superficie aproximada del $10 \%$ y se estima que aportan una escorrentía del $10 \%$ en invierno, y del $30-40 \%$ en verano. Por su parte, la precipitación es muy variable debido al efecto barrera de las altas cumbres, con valles interiores relativamente $\operatorname{secos}(<600 \mathrm{~mm} / \mathrm{año})$ y zonas de montañas con alta pluviosidad ( $>2.500 \mathrm{~mm} /$ año) (Clarvis et al., 2014). Los recursos hídricos que proporciona el río y sus afluentes permiten el desarrollo de usos económicos diversos como la agricultura, la industria química y el turismo. Pero sin duda, la energía 
hidroeléctrica es el factor socio-económico más importante, ya que el $56 \%$ de la energía consumida en Suiza proviene de estas plantas hidroeléctricas, lo que la convierte en un sector estratégico con el $80 \%$ del capital en manos de los cantones y municipios (www.swissinfo.ch).

Figura 1. Área de localización de la cuenca del río Ródano, y delimitación de los sistemas ambientales altitudinales.

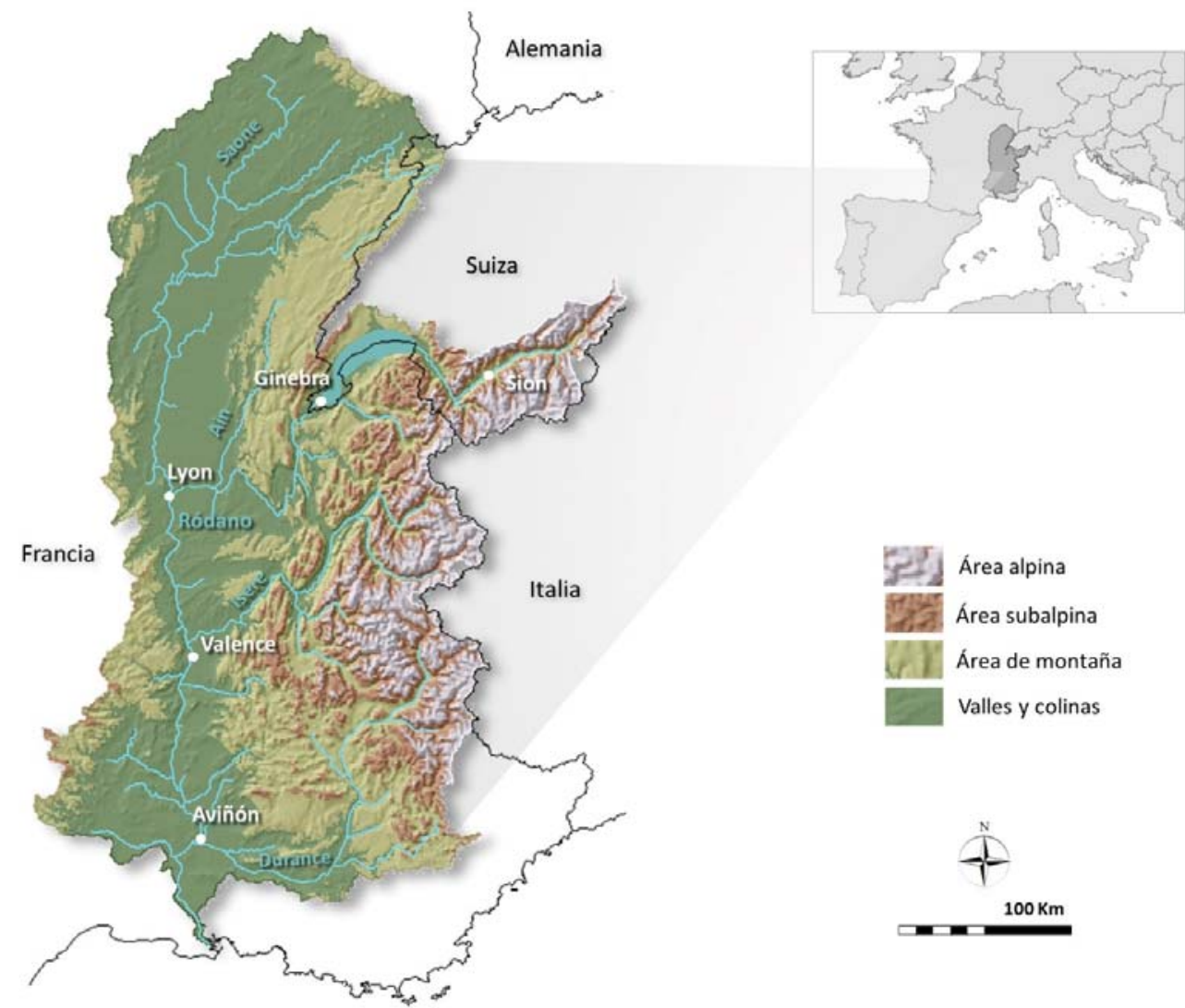

Fuente: Elaboración propia.

La segunda área de importancia concentra sus escorrentías en el Lago Ginebra. Con un área de $582 \mathrm{~km}^{2}$, sirve de frontera natural entre Francia y Suiza desde la Convención sobre la delimitación del límite en el lago el 25 de febrero de 1953 (Bréthaut y Clarvis, 2015). La gestión de esta lámina de agua posee un complejo reglamento de gestión en el que intervienen diferentes niveles institucionales de los estados de Francia y Suiza, incluida la legislación de la Unión Europea. Lo anterior permite la administración de diferentes infraestructuras que regulan el caudal del río. 
Y la tercera área queda definida por la vertiente francesa, en la que el río Ródano fluye hacia el sur hasta su desembocadura en el mar Mediterráneo. Esta parte del curso proporciona la mayor parte de los recursos económicos de toda la cuenca. En ella el río, apoyado por sus afluentes procedentes de los Alpes franceses (Iseré y Durance), sostienen una gran variedad de usos, entre los que destaca: el soporte a la producción de energía nuclear (cuatro centrales), el riego para los amplios valles agrícolas, el sector de la pesca fluvial, la producción de agua potable, el turismo, así como las necesidades de los ecosistemas de agua dulce, sin olvidar la producción de energía hidroeléctrica. Las funciones que desempeña el Ródano son de tal relevancia para la población que habita en sus riberas que ha propiciado la localización de números asentamientos humanos de grandes dimensiones sociodemográficas como las grandes ciudades de Lyon, Valence o Avignon. Desafortunadamente, al igual que sucede en gran parte de los cursos fluviales mediterráneos, el proceso de ocupación experimentado a lo largo de la historia presenta un sentido poco adaptativo, lo que ha generado importantes problemas por las recurrentes inundaciones. Las importantes avenidas producidas entre los años 2000 y 2003, llevaron a la implementación del 'Plan Rhone', un instrumento político destinado a gestionar el río de forma coordinada y evitar riesgos de inundación en la parte francesa de la cuenca (Guerrin, 2014).

Desde el punto de vista climático, la parte norte de la cuenca se encuentra bajo el dominio oceánico. Esta condición climática viene caracterizada por un régimen pluviométrico de fuertes lluvias durante el invierno que contrasta con la parte sur bajo la influencia mediterránea, de altas temperaturas en verano y fuertes lluvias en otoño y primavera. La margen oriental, de influencia alpina, proporciona un importante volumen de precipitación sólida en invierno, haciendo más complejo el régimen fluvial, con crecidas repentinas significativas que ocurren a finales de la primavera y en el comienzo del verano durante el deshielo (Etchevers et al., 2002).

De acuerdo a las proyecciones del cambio climático, en las próximas décadas el noroeste del ámbito mediterráneo de la cuenca podría experimentar un aumento de la temperatura del aire de $1 \mathrm{a} 3^{\circ} \mathrm{C}$ y una evolución incierta de las precipitaciones, sobre todo las invernales, con una posible variación de $\pm 10 \%$, y un descenso del $5 \%$ de los registros pluviométricos estivales. La influencia de estas alteraciones sobre la frecuencia de las inundaciones puede complicar la gestión de las actuales crecidas en el cauce bajo del Ródano y, con ello, la perdida de millones de euros por estos episodios (http://www.ird.fr/).

\section{Metodología}

El proceso metodológico se basó en la aplicación de un módulo hidrológico del modelo de servicios ecosistémicos InVEST (https://www.naturalcapitalproject.org/invest/), a través de su evaluación en cinco escenarios climáticos. El primero de ellos, referido al periodo actual, que sirvió de referencia en la comparación de dos escenarios de Concentración de Gases de Efecto 
Invernadero (RCP) en dos periodos futuros. Los resultados fueron cotejados en cuatro condiciones ambientales altitudinales para determinar la variabilidad espacial del balance de agua en la cuenca (Figura 2).

Figura 2. Esquema descriptivo del proceso metodológico.
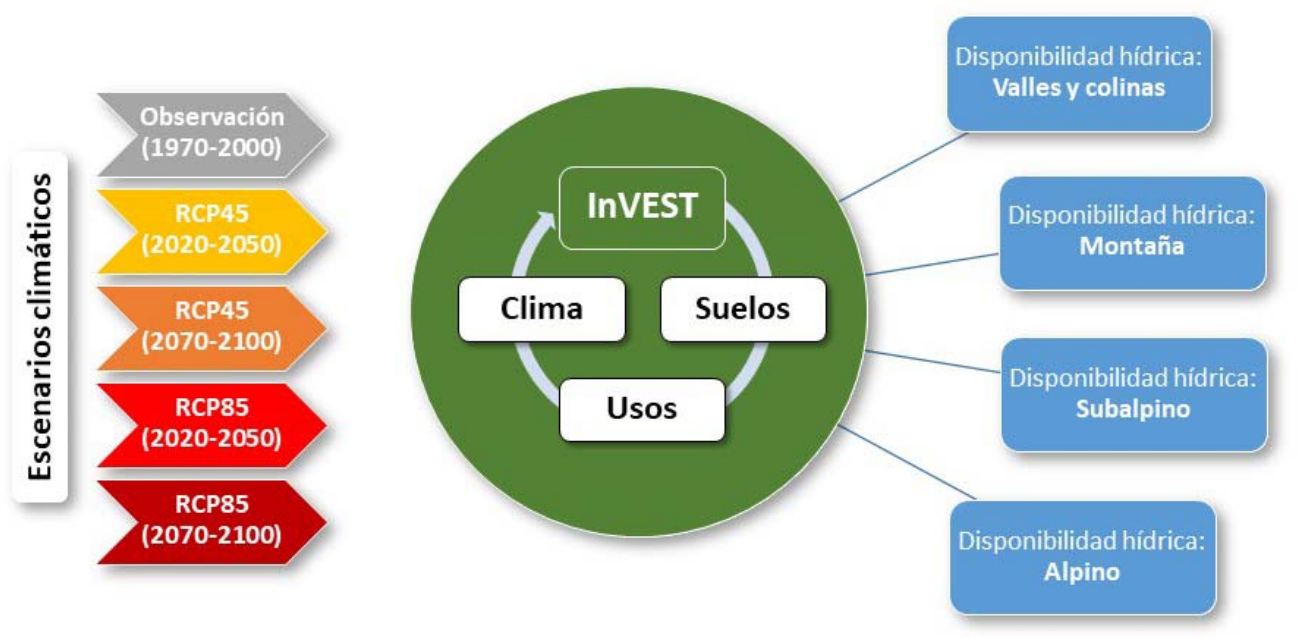

Fuente: Elaboración propia.

\subsection{Descripción del modelo 'Water Yield'}

Los cálculos realizados para la obtención de la disponibilidad de agua generada por la cuenca del Ródano se han llevado a cabo mediante el modelo 'Water Yield' del programa de análisis de servicios ecosistémicos InVEST v.3.3.3. De forma resumida, el modelo se basa en la estimación de las diferentes aportaciones desde cualquier punto de la cuenca teniendo en cuenta los cambios en los patrones de uso del suelo y su efecto al rendimiento hídrico superficial.

Los resultados estuvieron sujetos a un análisis espacial mediante algebra de mapas raster que se fundamentan en la estimación de la cantidad de agua captada por cada subcuenca en el área de trabajo. Con este valor se determina la cantidad de agua que fluye por cada píxel calculado en función de la precipitación y la evapotranspiración, por lo que toda el agua que no es evaporada se supone que representa el agua disponible. Por lo tanto, el modelo no diferencia entre la superficie, el subsuelo y el flujo de base, pero supone que todo el aporte de agua de un píxel alcanza un punto final de salida de la cuenca a través de una de estas vías. El proceso de cálculo estima el volumen de agua basándose en la curva de Budyko (propuestos por $\mathrm{Xu}$ et al., (2013)) y en la precipitación media anual. De manera simplificada se puede expresar como: 


$$
Y_{(x)}=\left(1-\frac{A E T_{(x)}}{P_{(X)}}\right) * P_{(x)}
$$

donde,

\author{
$Y_{(x)}$ : agua producida por año \\ $A E T_{(x)}$ : Evapotranspiración actual anual \\ $P_{(x)}$ : Precipitación anual
}

Por su parte, el efecto de las coberturas del suelo necesario para en el cómputo global se contempla mediante la consideración de las variables descritas en la tabla 1 . Estas son aplicadas sobre los algoritmos propuestos por $\mathrm{Fu}$ (1981) y Zhang et al., (2004) cuya formulación está disponible en http://data.naturalcapitalproject.org/nightly-build/invest-usersguide/html/reservoirhydropowerproduction.

Tabla 1. Valores de entrada y salida en el modelo 'Water Yield'de InVEST.

\begin{tabular}{ll}
\hline \multicolumn{1}{c}{ Requerimientos del modelo } & \multicolumn{1}{c}{ Salidas obtenidas } \\
\hline Mapa de usos (categórico) & Estimación de la fracción de ETr por Pr (pixel) \\
Delimitación de subcuencas & Estimación de la ETr $(\mathrm{mm} /$ pixel) \\
Profundidad de suelo $(\mathrm{mm})$ & Estimación de la disponibilidad de agua $(\mathrm{mm} / \mathrm{pixel})$ \\
Profundidad máx. de raíces por cobertura $(\mathrm{mm})$ & Pr media por pixel y cuenca $(\mathrm{mm})$ \\
Pr media anual $(\mathrm{mm})$ & ETP media por pixel y cuenca $(\mathrm{mm})$ \\
$\mathrm{ET}_{\mathbf{0}}$ media anual $(\mathrm{mm})$ & ETr media por pixel y cuenca $(\mathrm{mm})$ \\
Disponibilidad del contenido de agua por planta & Disponibilidad de agua por pixel y cuenca $(\mathrm{mm})$ \\
Coeficiente de ET $\mathrm{E}_{\mathbf{0}}$ por planta & Volumen de agua por cuenca $\left(\mathrm{m}^{3}\right)$ \\
Factor estacional & Volumen de agua en la cuenca por hectáreas $\left(\mathrm{m}^{3}\right)$ \\
\hline
\end{tabular}

${ }^{*}$ Evvapotranspiración de referencia $\left(\mathrm{ET}_{0}\right)$; Evapotranspiración real $(\mathrm{ETr})$; Evapotranspiración potencial (ETP); Precipitación (Pr).

Fuente: Elaboración propia, basado en Sharp et al., (2016).

\title{
3.2. Datos de entrada al modelo
}

\subsubsection{Coberturas del suelo}

Los usos del suelo se obtuvieron de la base de datos contenida en la Iniciativa para el Cambio Climático de la Agencia Espacial Europea (https://www.esalandcover-cci.org/). Dicha fuente aparece organizada en una cartografía global con 37 clases tipos, y una resolución espacial de $300 \mathrm{~m}$ por pixel.

\subsubsection{Delimitación de cuencas}

Los limites hidrológicos de la delimitación de la cuenca del Ródano se obtuvieron a partir del modelo digital del terreno SRTM DEM (https://www2.jpl.nasa.gov/srtm/) de $250 \mathrm{~m}$ de resolución de pixel, empleando el modelo hidrológico arcHydro Tools de 
arcGIS para la identificación espacial de las subcuencas. Los resultados fueron contrastados con los datos del programa HydroBASINS (Lehner y Grill 2013).

\subsubsection{Datos del suelo}

Para determinar el valor de profundidad de raíz de la vegetación se empleó la información de la base de datos SoilGrids (https://soilgrids.org) del Centro Internacional de Información y Referencia de Suelos (ISRIC). Con estos datos se pudo estimar la profundidad absoluta a la base de roca (en $\mathrm{mm}$ ) en formato raster y otro valor necesario, que fue la fracción de agua disponible para la planta (PAWF). En el catálogo de datos de SoilGrids se presenta un valor del contenido disponible de agua (AWC) a distintas profundidades, que servió para estimar la PAWF con la profundidad total del perfil de suelo. La resolución espacial para todas las capas de suelos es de $200 \mathrm{~m}$ por pixel.

\subsubsection{Datos climáticos}

Los datos climáticos se obtuvieron a través de dos fuentes. Por un lado, los valores históricos procedentes de la base de datos global (http://worldclim.org) en su segunda versión (Fick y Hijmans, 2017), con una resolución de $1 \mathrm{~km}$ por pixel. Éstos se emplearon como tiempo de observación y toman los registros promedio del periodo 1970-2000. Por otro, los correspondientes a los escenarios de cambio climático, los cuales proceden del Quinto Informe de Evaluación del Grupo Intergubernamental de Expertos sobre el Cambio Climático (IPCC) (2014). En este documento se trabaja sobre escenarios de emisiones que son proyectadas en estimaciones del cambio climático (van Vuuren et al., 2011) mediante un proceso de mejora de la escala ('downscaling' a $1 \mathrm{~km}$ de pixel) en una serie de 13 modelos climáticos regionales (http://www.euro-cordex.net/). Es evidente que estos cálculos tienen un elevado grado de incertidumbre, en gran medida debido a la dispersión entre las realizaciones de los distintos modelos climáticos, así como a la diversidad climática interna y a las dificultades para transferir los resultados del modelo climático a escalas espacial y temporal distintas. Para reducir esta variabilidad, se propuso elaborar un modelo único que asume los valores promedio del conjunto de modelos utilizados permitiendo aportar un sesgo reducido en los valores finales.

Las condiciones climáticas futuras propuestas, se basan en los Representative Concentration Pathways (RCP, Escenarios de Concentración de Gases de Efecto Invernadero), y se utilizan para producir un nuevo conjunto de simulaciones de modelos climáticos (Moss et al., 2010). En concreto, los análisis elaborados en este estudio aplican los RCP4.5 y RCP8.5 (que representan unas condiciones intermedias y extremas, respectivamente en el nivel de fuerza de radiación) proyectados para dos periodos, uno a corto plazo (valores promedio 2020-2050) y otro a medio plazo (valores promedio 2070-2100). La combinación de estas condiciones climáticas, resumidos en cuatro escenarios futuros, son definidos en adelante como: RCP45 2020-2050, RCP45 2070-2100, RCP85 2020-2050 y RCP85 2070-2100. 
En ambas bases de datos (histórico y escenarios futuros) se calculan los promedios anuales de precipitación, temperatura media, máxima y mínima, y radiación. Estos nuevos registros se emplean para estimar los valores de evapotranspiración de referencia $\left(\mathrm{ET}_{0}\right)$ espacialmente distribuidos, necesarios para el modelo 'Water Yield'. Para el cálculo de la $\mathrm{ET}_{0}$ se ha utilizado la fórmula de Hargreaves (Hargreaves y Samani, 1985). De forma simplificada:

\section{$E T_{0}=0,0023 *\left(T_{\text {med }}+17,78\right) * R_{0} *\left(T_{\text {max }}-T_{\text {min }}\right)$ \\ dónde:}

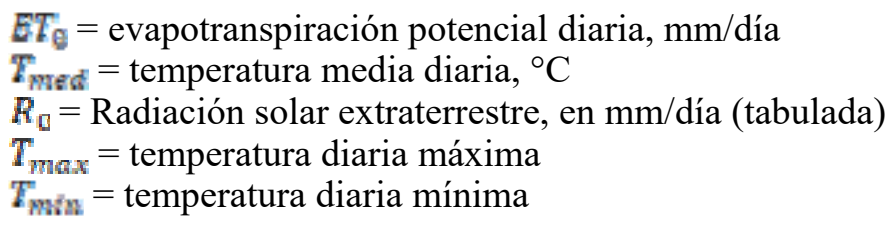

\subsection{Delimitación altitudinal de la Cuenca}

Para determinar unos límites que permitan mostrar espacios de características climáticas similares, se ha categorizado la cuenca en cuatro ámbitos espaciales de acuerdo a un criterio altitudinal (Figura 1). Los umbrales empleados se establecen de acuerdo a una clasificación fisionómica que contiene información sobre el rango altitudinal de especies vegetales (Becker et al., 2005), y que se organiza de la siguiente forma: a) valles y colinas $(<500 \mathrm{~m})$, superficie que acoge la mayor parte de las áreas agrícolas, en los que también se alternan los bosques de frondosas y el bosque mediterráneo, b) montaña (500-1200 m), espacios que se caracterizan por la existencia de importantes áreas de pastos para el ganado, y el desarrollo de bosques, principalmente mixtos y caducifolios, c) subalpino $(1200-2000 \mathrm{~m})$, formado los bosques de coníferas constituyendo las agrupaciones de masas forestales de mayor altitud y d) alpino (> $2000 \mathrm{~m}$ ), que reúne tres entornos, el primero más bajo (subnival), representado por una vegetación irregular restringida solo a los lugares más favorables y donde la nieve se encuentra durante parte del año. El segundo es el piso alpino, configurado por una alfombra cerrada de vegetación que incluye prados alpinos, arbustos esporádicos. Y por último, el piso glaciar, cubierto de nieve durante la mayor parte del año.

Los cambios en la cobertura vegetal pueden propiciar notables modificaciones sobre el ciclo del agua a escala de cuenca, por lo que esta clasificación contribuye a un mayor ajuste de la distorsión climática azonal que presentan las áreas de montaña. Este enfoque permite un análisis más pormenorizado a partir de una segmentación de los resultados del modelo en distintos sistemas ecológicos, y facilita la detección de los cambios en los patrones de la disponibilidad de agua en los escenarios de cambio climático propuestos. 


\section{Resultados}

\subsection{Tendencias de los datos mensuales de precipitación y evapotranspiración de referencia}

Como se ha descrito en el apartado de área de estudio, la cuenca del Ródano muestra, por su dimensión y heterogeneidad climática y orográfica, una alta complejidad en el análisis de sus factores físicos. El régimen pluviométrico presenta dos máximos que se producen en los periodos de primavera y otoño. No obstante, los meses de menor precipitación (julio y agosto) también reciben importantes aportaciones con más de 60 $\mathrm{mm}$ mensuales en promedio. Para el conjunto del año los valores medios son superiores a los $1000 \mathrm{~mm}$ (Figura 3a).

Figura 3. a) Eje izquierdo: valores mensuales de precipitación $(\mathrm{mm})$ para el periodo de observación (1970-2000); Eje derecho: tasa de variacion (\%) de los escenarios RCP respecto al periodo actual (observación). b) Eje izquierdo: valores mensuales de evapotranspiración de referencia $(\mathrm{mm})$ para el periodo de observación (1970-2000); Eje derecho: tasa de variacion (\%) de los escenarios RCP respecto al periodo actual (observación)

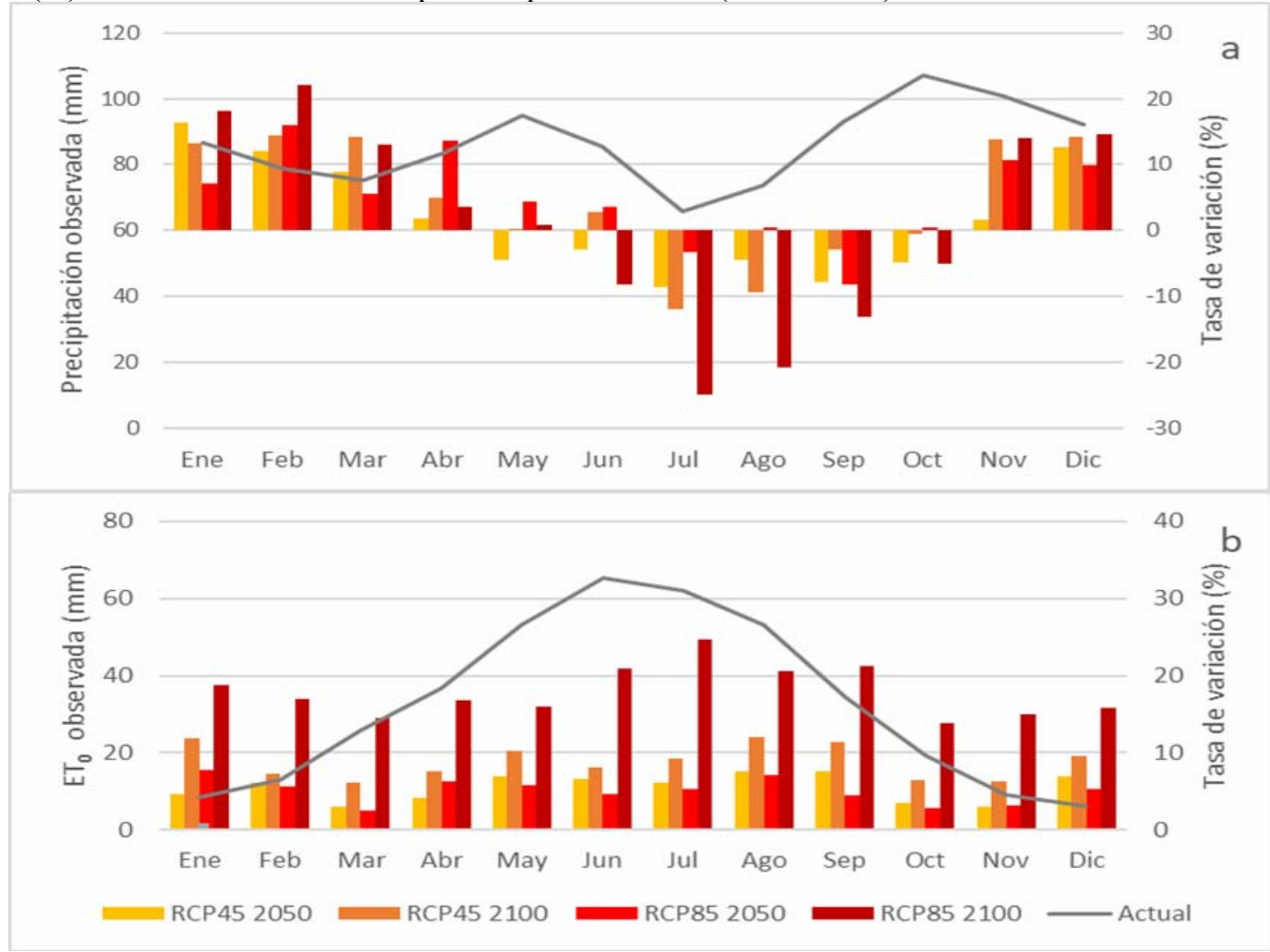

Fuente: Elaboración propia. 
Las tendencias de los escenarios de cambio climático estudiados ofrecen unos valores que indican importantes variaciones en la dinámica de la precipitación. De manera general, la variación en la lluvia estimada está relacionada con las diferentes condiciones de RCP y temporales; observado por el mayor nivel de concentración de gases de efecto invernadero y tiempo transcurrido, que incrementa la acentuación del efecto de cambio (Figura 3a). A este respecto, los resultados más interesantes se registran en la evolución mensual. En ellos se advierte un patrón de variación muy claro de forma estacional, con aumentos de la precipitación en el periodo otoñoinvierno y descensos muy pronunciados en primavera y verano, que en ambos casos pueden llegar a superar el $20 \%$.

Respecto a los datos de $\mathrm{ET}_{0}$, se observa una distribución más constante a lo largo del año, con un máximo en verano $(65 \mathrm{~mm})$ que desciende hasta los $6 \mathrm{~mm}$ de diciembre. Las tendencias de cambio futuras expresan un patrón similar en cada uno de los meses, con aumentos generalizados en las condiciones RCP45 para los dos periodos analizados. Mientras, para el RCP85, se observa una tendencia al alza más suave en el periodo 2020-2050, incremento que se dispara para el periodo 2070-2100 con subidas que superan el $15 \%$ (respecto a los valores históricos) en todos los meses y que llegan a niveles cercanos al $25 \%$ para los valores promedio del mes de julio (Figura 3b).

\subsection{Resultados del modelo 'Water Yield'}

Los principales resultados del modelo proporcionan una cuantificación espacial de los valores de la evapotranspiración real e hídrica de la cuenca. De esta información se obtienen los registros promedio para toda el área de estudio y los cinco escenarios. En estos últimos se observa un leve incremento de la precipitación en todos los casos en relación al valor de referencia actual (Figura 4). Respecto a la evapotranspiración potencial y real, no se advierten cambios significativos en los escenarios futuros, los cuales se mantienen en valores superiores a $315 \mathrm{~mm}$ en todos los casos, aunque apuntan un cierto aumento en los más desfavorables. 
Figura 4. Valores de precipitación, evapotranspiración de referencia, evapotranspiración real y la cuantificación hídrica de la cuenca, aportados por el modelo 'Water Yield'.

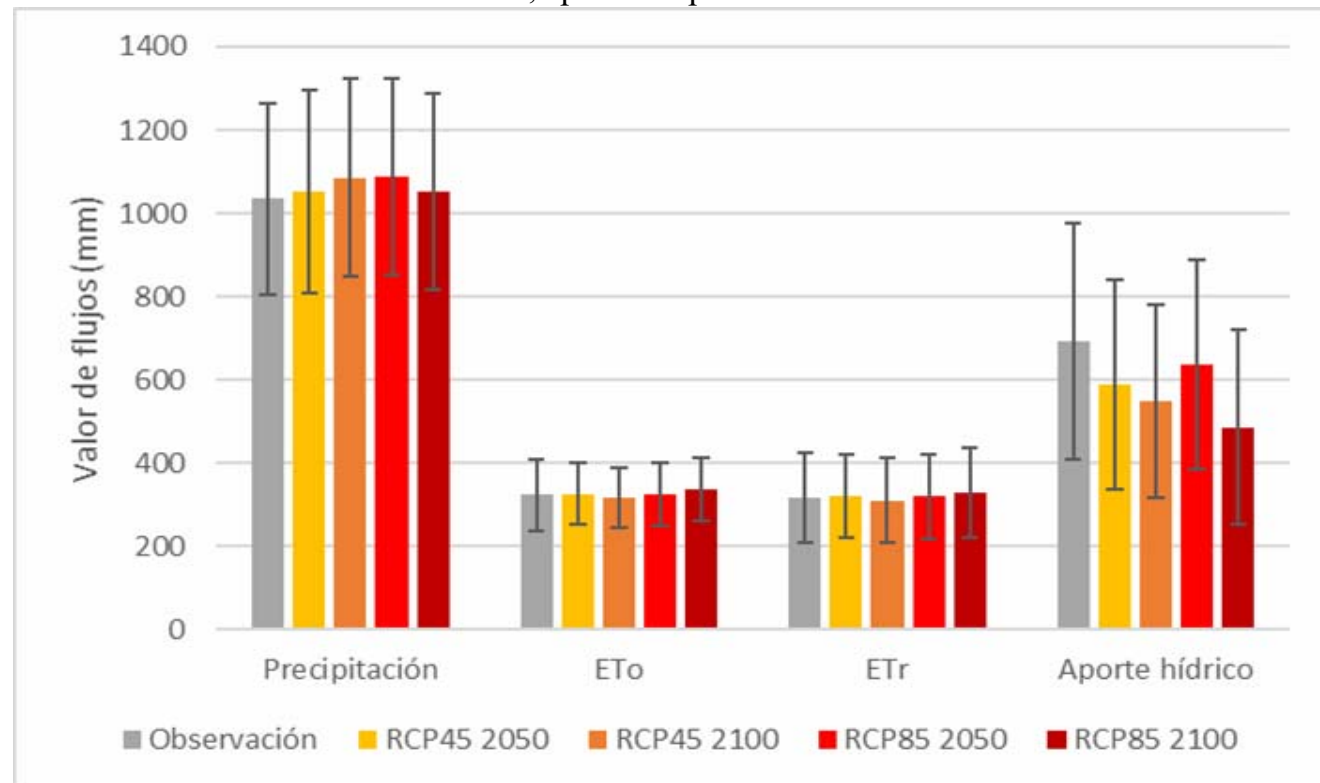

(*) Las barras muestran el aporte de los flujos en los distintos escenarios analizados. Los rangos superiores (en gris) muestran los datos de desviación estándar de los valores representados.

Fuente: Elaboración propia.

Por último, respectos a las estimaciones de agua generada por la cuenca, la tendencia indica un descenso destacable de la capacidad de producción hídrica en todos los escenarios de cambio climático. Pérdidas importantes, que llegan a ser más de $200 \mathrm{~mm}$ por pixel en el caso RCP85 del periodo 2070-2100, o lo que es lo mismo, una pérdida total de más de $20.000 \mathrm{Hm}^{3}$ en toda la cuenca que supondrá casi el $30 \%$ del total actual (Tabla 1).

Tabla 1. Cuantificación de los volúmenes del caudal de la cuenca en los cinco escenarios analizados. Y perdida de caudal de los escenarios RCP respecto al periodo actual (observación).

\begin{tabular}{lccc} 
& Volumen $\left(\mathbf{H m}^{\mathbf{3}}\right)$ & Perdida $\left(\mathbf{H m}^{\mathbf{3}}\right)$ & Perdida (\%) \\
\hline Escenario de observación & 67916,88 & --- & --- \\
RCP45 2020-2050 & 57761,13 & 10155,74 & 14,95 \\
RCP45 2070-2100 & 53698,85 & 14218,03 & 20,93 \\
RCP85 2020-2050 & 62361,60 & 5555,28 & 8,18 \\
RCP85 2070-2100 & 47624,12 & 20292,76 & 29,88 \\
\hline
\end{tabular}

Fuente: Elaboración propia. 


\subsection{Análisis altitudinal de los valores modelizados}

La distribución espacial de los valores modelizados de acuerdo a los criterios altitudinales previamente señalados, presenta variaciones significativas de los datos de precipitación, ETP y ETr. Además, proporciona con mayor claridad que áreas de la cuenca participan con mayor influencia en la disminución de los caudales que se han visto anteriormente a modo de conjunto del área de estudio.

Los gráficos de la figura 5 muestran las tasas porcentuales de cambio en las distintas variables analizadas respecto a los valores actuales. La precipitación (Figura 5a) ha presentado una correlación positiva con el criterio altitudinal, en la que se observó un ascenso progresivo en ambos periodos de RCP45 y para 2020-2050 del RCP85. El cambio fue homogéneo por escalones altitudinales, aunque es más acusado $(6 \%)$ en las zonas de valles y colinas $(>500 \mathrm{~m})$. Esta tendencia cambia en el RCP85 de 2070-2100, con un efecto notable en las áreas subalpinas (1200-2000 m) en las que el aumento es prácticamente nulo para este último escenario, llegando a invertirse en las zonas de montaña $(500-1200 \mathrm{~m})$ con la estimación de perdida de precipitación del $0,61 \%$.

Los valores de ETr responden de manera similar a la precipitación (Figura 5b), es decir, con pequeños incrementos para todos los escenarios futuros en todas las áreas altitudinales. Si bien, el efecto es más importante en las zonas alpinas $(>2000 \mathrm{~m})$, donde el aumento de la evapotranspiración llega a ser del 40, 58, 30 y $68 \%$ en los dos periodos de RCP45 y en los dos de RCP85, respectivamente (Figura 5b).

Por último, en lo que se refiere a los resultados del aporte hídrico de la cuenca fueron más variables (Figura 5c), proporcionando efectos muy diferentes dependiendo de las áreas altitudinales. En lo que respecta al primer escenario futuro (RCP45 2020-2050) se observa cómo se produjo un descenso de la disponibilidad de agua en todos los casos conforme se asciende en altitud, llegando a ser más del $8 \%$ en las áreas alpinas. Los RCP45 2070-2100 y RCP85 2020-2050 mostraron una gran heterogeneidad y resulta muy difícil interpretar una tendencia. Tanto para las áreas de valles y colinas, como para las áreas de montaña, se advirtió un incremento del aporte hídrico, mientras que desciende en las áreas subalpinas. El efecto es contrario en las superficies de mayor altitud, relativas a las áreas alpinas. Por último, el escenario más extremo (RCP85 2070-2100) mostró una tendencia de decrecimiento clara en todos los intervalos altitudinales, pero se traduce de manera más acentuada en las áreas de montaña y subalpinas con pérdidas del 11 y del $14 \%$, respectivamente (Figura $5 \mathrm{c}$ ). 
Figura 5. Tasas porcentuales de cambio en las distintas variables analizadas respecto a los valores actuales sistemas ambientales caracterizados por rasgos altitudinales $(<500$ : valles y colinas; 500-1200: montañas; 1200-2000: subalpino; $<2000$ : alpino). a) Tasas de cambio en la precipitación. b) Tasas de cambio en la evapotranspiración real. c) Tasas de cambio en los aportes hídricos.
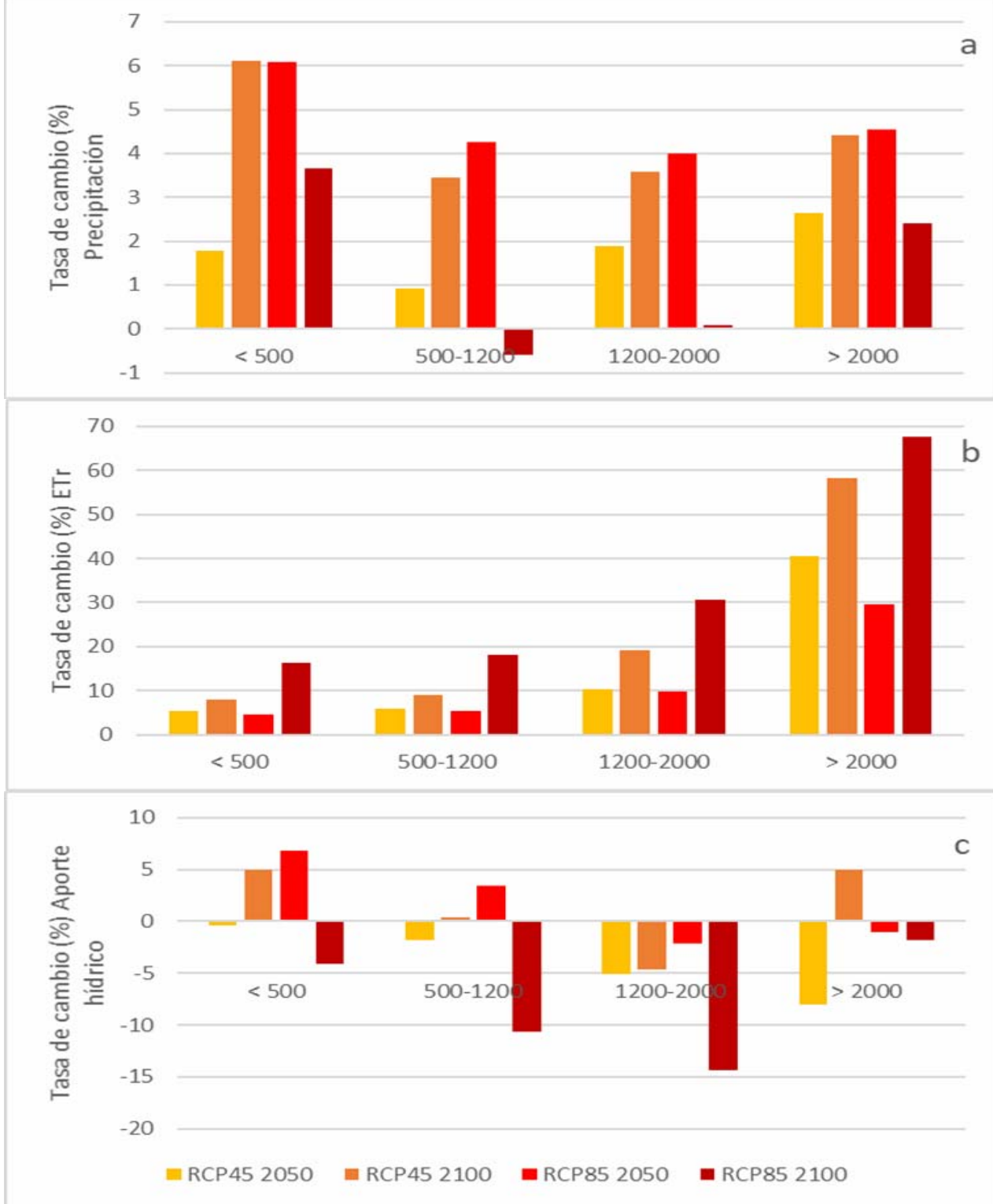

Fuente: Elaboración propia. 
En conjunto, para este último caso, se pudo determinar que las áreas que muestran un mayor incremento de la disponibilidad hídrica se da en las zonas de valle, mientras que de forma clara, las áreas subalpinas son las que experimentan las mayores pérdidas.

\section{Discusión}

La exploración de los datos de entrada al modelo 'Water Yield' de InVEST, expresados en valores mensuales de precipitación y $\mathrm{ET}_{\mathbf{0}}$, proporcionan una información muy interesante para determinar las tendencias en los escenarios de cambio climático futuros. Respecto a estos últimos, de manera indirecta relacionados con los datos de temperatura, muestran un incremento de sus valores con una intensidad similar a lo largo de todos los meses del año. Lo anterior repercute directamente, no solo a la perdida por evapotranspiración habitual en el periodo estival, sino que amplía este efecto durante el resto del año. Este cambio de tendencia afecta directamente al balance de agua de la cuenca lo que podría generar una pérdida considerable de alrededor de $100 \mathrm{~mm}$ cada $2{ }^{\circ} \mathrm{C}$ de aumento en la temperatura (Riedo et al., 2001).

La distribución mensual de la precipitación presenta unos resultados más llamativos, con una variación estacional mucho más significativa. Los escenarios de cambio climático elaborados muestran una fuerte disminución de la precipitación durante los meses más cálidos, factor que incide directamente en la actividad agropecuaria de la parte media y baja de la cuenca. Esta última podría verse especialmente afectada ya que está caracteriza por una gran diversidad donde predomina la producción lechera seguida de la viticultura y frutales (http://agreste.agriculture.gouv.fr/en-region/rhone-alpes/). A este respecto, la señalada disminución de la precipitación en los periodos de mayor productividad agrícola, unido al aumento de la evapotranspiración, parece que se empieza a manifestar de forma negativa en las comunidades agrícolas de montaña (Fuhrer et al., 2014), las cuales desempeñan un papel vital para las economías de estos valles y para el manteniendo el paisaje cultural alpino. Obviamente son las más vulnerables pues se enfrentan además a otras desventajas como baja productividad y altos costos de producción debido a la topografía, el suelo y restricciones climáticas (Mann, 2013).

A su vez, la variación de la precipitación descrita en los resultados, puede provocar un aumento de los valores actuales, que se evidencian entre los meses de noviembre y abril (Figura 3a), propiciando un reequilibrio en el balance anual de los registros. De esta forma, el régimen podría transformase de dos máximos a uno solo invernal y una estación seca estival muy acusada. Lógicamente, de materializarse esa tendencia, el régimen fluvial de la cuenca en las próximas décadas se vería especialmente alterado como así atestiguan otros resultados de modelos hidrológicos en trabajos recientes (Beniston et al., 2011; Chauveau et al., 2013; Boé and Habets 2014). Ante estas nuevas circunstancias climáticas, se prevé que el caudal circulante en superficie se reducirá significativamente a finales de siglo, provocando un 
descenso de la escorrentía en la mayoría de las subcuencas del Ródano, junto a una disminución en la frecuencia de inundaciones ordinarias y un aumento de la extraordinarias. (Ruiz-Villanueva et al., 2015).

En lo que se refiere a las posibles variaciones en el balance hídrico de la cuenca, los resultados presentan un patrón claro de perdida de volumen total de agua producida en ambos periodos y RCP estudiados. Se trata de una tendencia similar a la sufrida por el resto de cuencas del arco noroccidental mediterráneo, en los que Lespinas et al. (2010) determinan disminuciones significativas de un tercio de los caudales de las cuencas hidrográficas a finales de este siglo, que representan una reducción estimada del $20 \%$ de los recursos hídricos totales en esta región. Por lo tanto, si la evolución de los valores de precipitación y ETr, que expresan los datos de entrada y salida al sistema hídrico, no varían sustancialmente entre ellos (Figura 4), habrá que explorar otros posibles factores de la pérdida del caudal en la cuenca. En este sentido, se hace necesaria una revisión en detalle de los distintos espacios ecológicos de la cuenca que permitan obtener con mayor precisión como se ven afectados estos sistemas ambientales en distintos entornos altitudinales, y establecer cuál es el papel que desempeñan en el balance hídrico final de la cuenca.

En primer lugar, las zonas de valle y colinas, delimitadas por las superficies inferiores a la cota $500 \mathrm{~m}$, y ocupadas por las actividades agrícolas y bosques de frondosas, muestran los incrementos de precipitación más elevados (para RCP45 2070-2100 y RCP85 2020-2050 se estiman crecimientos del 6\%, lo que unido a la débil subida de la evapotranspiración podría suponer una mayor disponibilidad de recursos hídricos superior, al 5\%. En los análisis llevados a cabo por Etchevers et al. (2002) la respuesta de la escorrentía varía por subcuencas hidrográficas según las anomalías meteorológicas y las características intrínsecas de cada zona (suelo, vegetación y clima dominante), pero en general aportan valores similares a los del presente trabajo. De forma consistente a los resultados obtenidos, los valles fluviales del norte, relativamente más lluviosos (Saone, Doubs y Ain), la precipitación y la escorrentía aumentan para el periodo de análisis (2054-2064) entre 17-29 \% y 20$48 \%$, respectivamente, mientras que el aumento de la evaporación es relativamente débil $(+13 \%)$. En cambio, en la parte meridional estos datos se reducen, pero parecen estar más asociados al descenso de la escorrentía que pasa a niveles más bajos de agua subterránea y a unas temperaturas mayores que favorecen la infiltración en estos suelos menos húmedos (Lespinas et al., 2010).

Por su parte, en los ambientes situados en el ámbito comprendido entre las cotas 500-1200, y el paisaje en el tercer nivel (1200-2000 m) las salidas del modelo proporcionan resultados con patrones de cambio similares. Es significativo el descenso de la capacidad de producción hídrica de estos espacios, sobre todo en las altitudes mayores y de forma muy pronunciada en el escenario más extremo (RCP85 2070-2100). Estas áreas intermedias son muy susceptibles a los cambios provocados por la alteración del clima en los escenarios futuros, variaciones que están fuertemente influidas por la transformación de las coberturas de suelo debido a los cambios de usos (Rahman et al., 2015). La subida generalizada de las temperaturas, mostradas ya por los valores del escenario de observación, favorece la expansión de 
espacios propicios para el desarrollo tanto de los cultivos como de la vegetación natural en cotas más elevadas. Estudios recientes han detectado que durante las últimas décadas se ha producido una tendencia general en el aumento de la cubierta vegetal (greening up) en las montañas de la cuenca norte mediterránea que está provocando alteraciones en el ciclo del agua (Farley et al., 2005, Molina et al., 2015). El aumento de la masa forestal en áreas como la de estudio, donde de partida existe una importante cobertura vegetal, puede generar una reducción sustancial de la escorrentía (Moran-Tejada et al., 2010; López-Moreno et al., 2008; Pérez-Cutillas et al., 2018) vinculada a la mejora de la estructura de los suelos y aumento de su capacidad de infiltración. Con ello se podría explicar la perdida de parte de los flujos de agua, ya que un crecimiento de esta superficie vegetal también lleva asociado un efecto de partición que introduce la vegetación a través de procesos como la evaporación, transpiración, e interceptación, con una nueva redistribución de la precipitación (García-Ruiz et al., 2011, Linhoss y Siegert 2016).

Finalmente, el último conjunto ambiental situado en la parte oriental de la cuenca es el asociado al nivel superior $(>2000 \mathrm{~m})$ de paisaje puramente alpino. Los resultados del modelo proporcionan un leve incremento de la precipitación en todos los escenarios que siguen la tendencia del resto de niveles altitudinales. Pero el efecto que más claramente se observa en estas áreas de mayor altitud es el incremento más elevado de la evapotranspiración con tasas de cambio entre el 30 y el $68 \%$, dependiendo del escenario (Figura 5b). El aumento del albedo superficial provocado por la pérdida de una gran superficie de nieve ejerce una influencia local en la atmósfera y en la hidrología a través del mecanismo de retroalimentación que acrecienta la fusión del hielo y el retroceso de los glaciares (Fassnacht et al., 2016). Este proceso acelerado de perdida de las capas permanentes de nieve puede explicar una parte importante de la perdida de aporte de agua en alta montaña, donde tres de los cuatro escenarios analizados muestran valores negativos respecto al escenario actual. Estos datos coinciden con otros estudios llevados a cabo en estos entornos alpinos, los cuales concluyen que son los espacios más sensibles y proclives a sufrir las mayores transformaciones provocadas por los efectos del cambio climático. En dichos estudios se ha estimado que en los próximos 100 años los glaciares alpinos pueden perder entre $50 \%$ y $90 \%$ de su volumen actual, y por cada grado de subida de la temperatura, la cota promedio de nieve aumentará en $150 \mathrm{~m}$ de la altitud a la que se encuentra en la actualidad (Beniston, 2012). Los sistemas hidrológicos, directamente conectados a los procesos de hielo y deshielo glaciar, responderán en cantidad y estacionalidad a precipitaciones de alta variabilidad e incertidumbre, pero con un patrón claro de mayor riesgo de inundación durante la primavera, y un aumento de las sequías en verano y otoño (Huss et al., 2014, Kormann et al., 2015).

\section{Conclusiones}

Las principales conclusiones que se pueden extraer de este trabajo atañen a los resultados obtenidos de la aplicación del modelo hidrológico. En primer lugar, se 
observa una reducción significativa del caudal de la cuenca en ambos periodos y RCP estudiados respecto al valor de referencia actual. Los incrementos en la precipitación y el mantenimiento de la $\mathrm{ET}_{0}$ no explican esta perdida en la dinámica del balance del agua, lo que orienta la investigación hacia otros factores de redistribución de la precipitación como son los cambios en las coberturas del suelo.

El análisis de los diferentes sistemas ambientales caracterizados por rasgos altitudinales, establecen importantes diferencias en el balance hídrico final en el conjunto de la cuenca. El efecto que más claramente se advierte se da en las áreas alpinas $(>2000 \mathrm{~m})$ con aumentos de la evapotranspiración que en algunos casos se acerca al $70 \%$, y que podrían estar asociados al incremento del albedo por la pérdida de la cobertura glaciar. Este proceso acelerado de perdida de las capas permanentes de nieve puede explicar una parte importante de la disminución del caudal aportado por las altas cumbres.

Los resultados alcanzados pueden ser extrapolados a otras áreas próximas de la cuenca mediterránea, donde la pérdida paulatina de los recursos que indican los modelos supondrá importantes adaptaciones a nuevos desafíos impuestos por estos escenarios de escasez de agua. El desarrollo de herramientas que permitan conocer los cambios de los elementos que componen el ciclo hidrológico podrán servir de base para las soluciones relacionadas con la gestión del agua y sus demandas futuras, así como en la elaboración de políticas más efectivas en la administración de los recursos hídricos.

\section{Bibliografía}

Barboux, C., Strozzi, T., Delaloye, R., Wegmüller, U. y Collet, C. (2016): Mapping slope movements in Alpine environments using TerraSAR-X interferometric methods. ISPRS Journal of Photogrammetry and Remote Sensing, 109, 178-192.

Becker, T., Dietz, H., Billeter. R., Buschmanna, H. y Edwards, P.J. (2005): Altitudinal distribution of alien plant species in the Swiss Alps. Perspectives in Plant Ecology, Evolution and Systematics, 7 (3), 173-183.

Beniston, M., Stoffel, M. y Hill, M. (2011): Impacts of climatic change on water and natural hazards in the Alps: can current water governance cope with future challenges? examples from the European "ACQWA" project. Environ Sci Policy, 14 (7), 734-743. doi:10.1016/j.envsci.2010.12.009

Beniston, M. (2012): Impacts of climatic change on water and associated economic activities in the Swiss Alps. Journal of Hydrology, 412-413, 291-296.

Beniston M. y Stoffel M. (2014): Assessing the impacts of climatic change on mountain water resources. Sci Total Environ, 493, 1129-1137. doi:10.1016/j.scitotenv.2013.11.122

Bethoux, J.P. y B. Gentili. (1999): Functioning of the Mediterranean Sea: Past and present changes related to freshwater input and climate changes, J. Mar. Syst., 20, 33- 47.

Boé, J. y Habets, F. (2014): Multi-decadal river flow variations in France. Hydrol Earth Syst Sci, 18, 691-708. 
Bréthaut, C. y Clarvis, M.H. (2015): Interdisciplinary approaches for analysing governance challenges across the Rôhne basin. Reg Environ Change, 15, 499-503.

Bréthaut, C. y Pflieger, G. (2015): The shifting territorialities of the Rhone River's transboundary governance: a historical analysis of the evolution of the functions, uses and spatiality of river basin governance. Reg Environ Change, 15, 549-558. DOI 10.1007/s10113-013-0541-4

Chauveau, M., Chazot, S., Perrin, C., Bourgin, P.Y., Sauquet, E., Vidal J.P., Rouchy, N., Martin, E. David, J., Norotte, T., Maugis, P. y De Lacaze, X. (2013): Quels impacts des changements climatiques sur les eaux de surface en France à l'horizon 2070? La Houille Blanche - Revue internationale de l'eau, EDP Sciences, 5-15.

Clarvis, M.H., Fatichi, S., Allan, A., Fuhrer, J., Stoffel, M., Romerio, F., Gaudard, L., Burlando, P., Beniston, M., Xoplaki, E. y Toreti, A. (2014): Governing and managing water resources under changing hydroclimatic contexts: the case of the upper Rhone basin. Environ Sci Policy, 43, 56-67. doi:10.1016/j.envsci.2013.11.005

Delaloye, R., Lambiel, C. y Gärtner-Roer, I. (2010): Overview of rock glacier kinematics research in the Swiss Alps: Seasonal rhythm, interannual variations and trends over several decades. Geographica Helvetica, 65 (2), 135-145.

Etchevers, P., Golaz, C., Habets, F. y Noilhan, J. (2002): Impact of a climate change on the Rhone river catchment hydrology. Journal of Geophysical Research, 107 (D16), 6-18. doi:10.1029/2001JD00049

Farley, K.A., Jobbágy, E.G. y Jackson, R.B. (2005): Effects of afforestation on water yield: A global synthesis with implications for policy. Global Change Biology 11, 1565-1576. doi: 10.1111/j.1365-2486.2005.01011.x

Fassnacht, S.R., Cherry, M.L., Venable, N.B.H. y Saavedra, F. (2016): Snow and albedo climate change impacts across the United States Northern Great Plains. Cryosphere, 10 (1), 329-339. doi: 10.5194/tc-10-329-2016

Fatichi, S., Rimkus, S., Burlando, P. y Bordoy, R. (2014): Does internal climate variability overwhelm climate change signals in streamflow? The upper Po and Rhone basin case studies. Science of the Total Environment, 493, 1171-1182.

Fick, S.E. y Hijmans, R.J. (2017): Worldclim 2: New 1-km spatial resolution climate surfaces for global land areas. International Journal of Climatology. DOI: 10.1002/joc.5086

Fu, B. P. (1981): On the calculation of the evaporation from land surface, Sci. Atmos. Sin., 5, 23-31.

Fuhrer, J., Smith, P. y Gobiet, A. (2014): Implications of climate change scenarios for agriculture in alpine regions - A case study in the Swiss Rhone catchment. Science of the Total Environment, 493, 1232-1241.

García-Ruiz, J.M., López-Moreno, J.I., Vicente-Serrano, S.M., Lasanta-Martínez, T. y Beguería, S. (2011): Mediterranean water resources in a global change scenario. EarthScience Reviews, 105, 121-139. doi: 10.1016/j.earscirev.2011.01.006

Gaudard, L., Romerio, F., Dalla Valle, F., Gorret, R., Maran, S., Ravazzani, G., Stoffel, M. y Volonterio, M. (2014): Climate change impacts on hydropower in the Swiss and Italian Alps. Sci Total Environ, 493, 1211-1221. doi:10.1016/j.scitotenv.2013.10.012 
Gobiet, A., Kotlarski, S., Beniston, M., Heinrich, G., Rajczak, J. y Stoffel, M. (2014): 21st century climate change in the European Alps - A review. Science of the Total Environment, 493, 1138-1151.

Guerrin, J. (2014): A floodplain restoration project on the River Rhône (France): analyzing challenges to its implementation. Regional Environmental Change, 15 (3), 559-568. doi:10.1007/s10113-014-0650-8

Hargreaves, G.H. y Samani, Z.A. (1985): Reference crop evapotranspiration from temperature. Applied Eng. in Agric., 1 (2), 96-99.

Huss, M., Zemp, M., Joerg, P.C. y Salzmann, N. (2014): High uncertainty in 21st century runoff projections from glacierized basins. Journal of Hydrology, 510, 35-48.

IPCC, (2014): Climate Change 2014: Synthesis Report. Contribution of Working Groups I, II and III to the Fifth Assessment Report of the Intergovernmental Panel on Climate Change [Core Writing Team, R.K. Pachauri and L.A. Meyer (eds.)]. IPCC, Geneva, Switzerland, $151 \mathrm{pp}$.

Kormann, C., Francke, T. y Bronstert, A. (2015): Detection of regional climate change effects on alpine hydrology by daily resolution trend analysis in Tyrol, Austria. Journal of Water and Climate Change, 6 (1), 124-143. doi: 10.2166/wcc.2014.099

Lehner, B. y Grill G. (2013): Global river hydrography and network routing: baseline data and new approaches to study the world's large river systems. Hydrological Processes, 27(15), 2171-2186. Datos disponibles en: www.hydrosheds.org.

López-Moreno, J. I., Vicente-Serrano, S. M., Zabalza, J., Revuelto, J., Gilaberte, M., AzorínMolina, C., Morán-Tejeda, E., García-Ruiz, J. M. y Tague, C. (2014): Respuesta hidrológica del Pirineo central al cambio ambiental proyectado para el siglo XXI. Pirineos, 169, e004. doi: http://dx.doi.org/10.3989/Pirineos.2014.169004

Mann, S. (2013): The future of mountain agriculture. En: Mann S, editor. Heidelberg: Springer Verlag.

Mendizabal, M., Sepúlveda, J. y Torp, P. (2014): Climate change impacts on flood events and its consequences on human in Deba River. International Journal of Environmental Research, 8 (1), 221-230.

Molina, A., Vanacker, V., Brisson, E., Mora, D. y Balthazar, V. (2015): Multidecadal change in streamflow associated with anthropogenic disturbances in the tropical Andes. Hydrology and Earth System Sciences, 19, 4201-4213. doi: 10.5194/hess-19-4201-2015

Morán-Tejeda, E., Ceballos-Barbancho, A. y Llorente-Pinto, J.M. (2010): Hydrological response of Mediterranean headwaters to climate oscillations and land-cover changes: The mountains of Duero River basin (Central Spain). Global and Planetary Change, 72, 39-49. doi: 10.1016/j.gloplacha.2010.03.003

Moss, R.H., Edmonds, J.A., Hibbard, K.A., Manning, M.R., Rose, S.K., van Vuuren, D.P., Carter, T.R., Emori, S., Kainuma, M., Kram, T., Meehl, G.A., Mitchell, J.F.B., Nakicenovic, N., Riahi, K., Smith, S.J., Stouffer, R.J., Thomson, A.M., Weyant, J.P. y Wilbanks, T.J., (2010): The next generation of scenarios for climate change research and assessment. Nature, 463 (7282), 747-756.

Lamouroux, N., Olivier, J.M., Camenen, B., Castella, E., Clemens, A. Doledec, S., Forcellini. M., Piégay, H., Riquier, J. y Rosset, V. (2013): RhônEco (1998-2018). Suivi écologique de 
la restauration du Rhône. Bilan et Proposition technique pour la période 2014-2018. [Rapport de recherche] ZABR, Lyon. 2013, 9 p.

Lespinas, F., Ludwig, W. y Heussner, S. (2010): Impact of recent climate change on the hydrology of coastal Mediterranean rivers in Southern France. Climatic Change, 99, 425456. doi: 10.1007/s10584-009-9668-1

Linhoss, A.C. y Siegert, C.M. (2016): A comparison of five forest interception models using global sensitivity and uncertainty analysis. Journal of Hydrology, 538, 109-116. doi: 10.1016/j.jhydrol.2016.04.011

Pavanelli, D. y Capra, A. (2014): Climate change and human impacts on hydroclimatic variability in the reno river catchment, Northern Italy. Clean - Soil, Air, Water, 42 (5), 535-545.

Pérez-Cutillas, P., Zema, D.A., Cataldo, M.F., de Vente, J. y Boix-Fayos, C. (2017): Efectos de la revegetación a escala de cuenca sobre el caudal y la evapotranspiración en ambiente mediterráneo. Cuenca del Taibilla (SE de España). Bosque, 39 (1), (en prensa).

Pellicciotti, F., Carenzo, M., Bordoy, R. y Stoffel, M. (2014): Changes in glaciers in the Swiss Alps and impact on basin hydrology: current state of the art and future research. Sci Total Environ, 493, 1152-1170. doi:10.1016/j.scitotenv.2014.04.022

Rahman, K., Gago da Silva, A., Moran Tejeda, E., Gobiet, A., Beniston, M. y Lehmann, A. (2015): An independent and combined effect analysis of land use and climate change in the upper Rhone River watershed, Switzerland. Applied Geography, 63, 264-272.

Riedo, M., Gyalistras, D. y Fuhrer, J. (2001): Pasture responses to elevated temperature and doubled CO2 concentration: assessing the spatial pattern across an alpine landscape. Clim Res, 17, 19-31.

Rohling, E.J. y Bryden, H.L. (1992): Man-induced salinity and temperature increases in Western Mediterranean Deep Water, J. Geophys. Res., 97, 191-198.

Ruiz-Villanueva, V., Stoffel, M., Bussi, G., Francés, F. y Bréthaut, C. (2015): Climate change impacts on discharges of the Rhone River in Lyon by the end of the twenty-first century: model results and implications. Reg Environ Change, 15, 505-515. doi: 10.1007/s10113014-0707-8

Sharp, R., Tallis, H.T., Ricketts, T., Guerry, A.D., Wood, S.A., Chaplin, Kramer, R., Vogl, A. L. (2016): InVEST User Guide. Stanford. Disponible en: http://ncpdev. stanford.edu/ dataportal/invest]releases/

documentation/current_release/index.html

Tonka, L. (2014): Hydropower license renewal and environmental protection policies: a comparison between Switzerland and the USA. Regional Environmental Change, 15 (3), 539-548. doi:10.1007/s10113-014-0598-8

van Vuuren, D., Edmonds, J., Kainuma, M., Riahi, K., Thomson, A., Hibbard, K., Hurtt, G., Kram, T., Krey, V., Lamarque, J.-F., Masui, T., Meinshausen, M., Nakicenovic, N., Smith, S. y Rose, S. (2011): The representative concentration pathways: an overview. Climatic Change, 109(1), 5-31.

Vivrioli, D. y Weingartner, R. (2008): Water towers a global viewon the hydrological importance of mountains. En: Wiegandt E, editor. Mountains: sources ofwater, sources of knowledge. Adv Global Change ResDordrecht: Springer-Verlag. p. 15-20. 
Xu, X., Liu, W., Scanlon, B. R., Zhang, L., y Pan, M. (2013): Local and global factors controlling water-energy balances within the Budyko framework. Geophysical Research Letters, 40 (23), 6123-6129. doi:10.1002/2013GL058324.

Zhang, L., Hickel, K., Dawes, W.R., Chiew, F.H.S., Western, A.W. y Briggs, P.R. (2004): A rational function approach for estimating mean annual evapotranspiration. Water Resources Research, 40 (2). DOI: 10.1029/2003WR002710.

\section{Agradecimientos}

Este trabajo ha sido desarrollado en el marco del proyecto de investigación 'Identification of realistic scenarios of climate change and Sustainable Land Management (SLM) for 2010-2100 period, in the catchment area of the Mediterranean Sea', financiado por la Universidad de Friburgo (Suiza) en el programa de becas de internacionalización del periodo 2016-2017. Mencionar de manera especial a los investigadores Joris de Vente y Carolina Boix-Fayos del Grupo de Erosión y Conservación de Suelos del Centro de Edafología y Biología Aplicada del Segura (CEBAS-CSIC), y al profesor Reynald Delaloye del grupo de Geografía Física del Departamento de Geociencias (Universidad de Friburgo) por su ayuda y apoyo en el desarrollo del proyecto. 